\title{
Estabilidade do Estado Homogêneo em Redes de Populações Acopladas
}

J.A.L. SILVA ${ }^{1}$ M.L. CASTRO, D.A.R. JUSTO, Departamento de Matemática Pura e Aplicada, Universidade Federal do Rio Grande do Sul, Av. Bento Gonçalves 9500, Porto Alegre, RS, Brasil.

Resumo. Nos últimos anos tem havido um crescente interesse pelo estudo da dinâmica de metapopulações. Há vários estudos visando demonstrar que a fração migratória não influencia na estabilidade do equilíbrio homogêneo. Neste trabalho obtemos uma versão completa deste resultado, incorporando a possibilidade de movimento dependente da densidade populacional local. Os resultados obtidos nos permitem concluir que para frações migratórias constantes, a estabilidade do modelo local é preservada, porém a metodologia usada nos permite construir exemplos onde a dependência da densidade local na fração migratória pode tornar o equilíbrio homogêneo instável mesmo que o sistema local desacoplado apresente estabilidade.

\section{Introdução}

Na última década foi notável o crescimento da pesquisa em modelos de dinâmica populacional com estrutura espacial. As revisões apresentadas em $[5,8]$ fornecem uma boa amostra do interesse pelo estudo de modelos de metapopulações. Uma prática bastante comum na modelagem de populações distribuídas em habitats fragmentados é o uso de modelos espacialmente explícitos. Nestes modelos a população, neste caso dita metapopulação, é tratada como um conjunto discreto de subpopulações localizadas em fragmentos de habitat que são adequados para a reprodução e sobrevivência. A conexão desses fragmentos é feita através de movimentos migratórios entre as populações locais mais próximas.

Vários trabalhos destacam a importância do movimento migratório como causador de efeitos estabilizadores e simplificadores na dinâmica da metapopulação [12, 16, 3, 22]. Em particular Rohani et al. [20] demonstraram que para uma metapopulação de uma única espécie com taxa migratória constante entre os fragmentos mais próximos o movimento migratório não tem nenhuma influência sobre a estabilidade do estado homogêneo. Ruxton [21] considerou a possibilidade da migração depender da densidade populacional local mas mesmo assim encontrou poucos indícios de que a dispersão pudesse causar efeitos desestabilizadores.

Neste trabalho propomos um tratamento mais geral e mais rigoroso para este tipo de modelo incluindo a possibilidade de dispersão dependente da densidade

${ }^{1}$ jaqx.mat.ufrgs.br 
local. Além disso, os resultados obtidos permitem concluir que a dispersão pode ser causadora de instabilidades em certas circunstâncias.

\section{O Modelo}

Considere uma coleção de $n$ fragmentos de habitats adequados para uma certa espécie. Cada um desses fragmentos está cercado por um meio hostil e totalmentente inadequado para a reprodução e sobrevivência desta espécie. Em cada um desses fragmentos vive uma população desta espécie. Vamos supor por simplicidade que as gerações, sempre tomadas em tempos discretos $t=0,1,2, \ldots$ jamais se entrelacem. Vários exemplos de populações naturais se enquadram nesta formulação [8].

Inicialmente vamos imaginar que não há conexão entre os sítios (fragmentos). Cada subpopulação floresceria de maneira independente das demais. Se denotamos por $x_{t}$ o número de indivíduos num desses sítios no instante $t$, então a dinâmica da subpopulação deste sítio é dada por

$$
x_{t+1}=f\left(x_{t}\right),
$$

onde $f$ é uma função suave. A equação acima incorpora os processos de reprodução e mortalidade de geração a geração de modo que estes processos dependem da densidade populacional do fragmento em questão através da função $f$. Vamos supor que $f$ possua um único ponto fixo positivo, $\bar{x}$, isto é,

$$
\bar{x}=f(\bar{x}), \bar{x}>0 .
$$

A equação (2.1) pode ser linearizada em torno do equilíbrio $\bar{x}$ resultando no critério de estabilidade

$$
\begin{gathered}
\left|f^{\prime}(\bar{x})\right|<1 \rightarrow \bar{x} \text { estável, } \\
\left|f^{\prime}(\bar{x})\right|>1 \rightarrow \bar{x} \text { instável. }
\end{gathered}
$$

A dinâmica da equação (2.1) já foi alvo de vários estudos. Fenômenos de grande interesse como cascata de bifurcações e caos já foram observados para várias escolhas de mecanismos representando a dependência da densidade, isto é, escolhas para a função $f[17,18,19,6]$. Talvez o mais célebre destes exemplos seja o da aplicação logística (ver Figura 1):

$$
f(x)=r x(1-x), \quad 0<r \leq 4, \quad 0 \leq x \leq 1 .
$$

Agora vamos estabelecer ligações entre as subpopulações. A cada geração um processo migratório ocorre entre os sítios. Para cada sítio, uma fração $\mu$ de indivíduos deixa o dado sítio e migra para os sítios mais próximos. Certos aspectos devem ser classificados:

I O processo migratório é de curta duração (comparado à escala de tempo usada no modelo que é o tempo entre duas gerações consecutivas) e portanto é razoável supor que o movimento migratório é $100 \%$ bem sucedido, isto é, não ocorrem mortes durante o movimento. Além disso, ocorre apenas um movimento migratório a cada geração. 


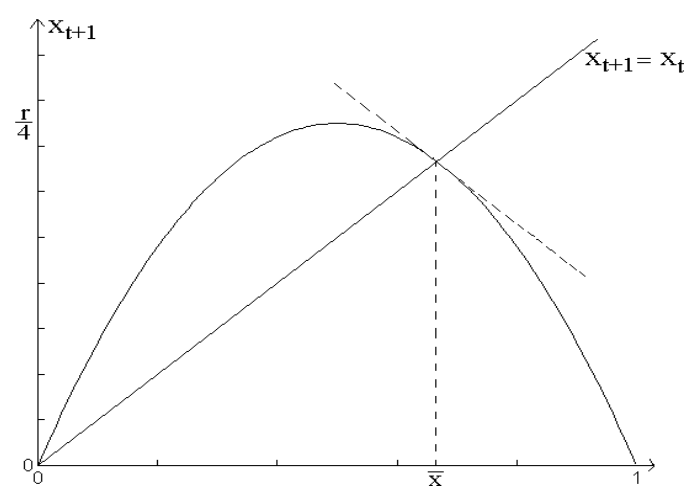

Figura 1: Gráfico de $x_{t+1}=r x_{t}\left(1-x_{t}\right)$. A estabilidade do ponto fixo $\bar{x}$ depende da declividade da linha pontilhada conforme equação (2.3).

II Deve ser estabelecida uma ordem para os eventos: migração e dinâmica local (reprodução e mortalidade). A falha na separação destes dois processos integralmente distintos pode acarretar em resultados fantasiosos (ver [10]).

III A fração migratória $\mu$ de indivíduos que deixam uma dado sítio pode depender da densidade local (sítio em questão ) e também da densidade dos sítios vizinhos (canditatos a receptores dos imigrantes). No presente artigo apenas a dependência local será incluída no modelo. Assim, supomos que $\mu$ é uma função crescente e de classe $C^{1}$. Além disso, por ser uma proporção, $0 \leq \mu(x) \leq 1, \forall x \geq 0$. Para exemplos ver [21].

IV A topologia deve ser definida, isto é, devemos estabelecer quem é vizinho de quem. Metapopulações naturais podem ter topologias bastante complexas (ver [8]) já que não apenas a proximidade geográfica é relevante mas também a capacidade de movimento entre 2 sítios quaisquer. Vamos supor que as vizinhanças são simétricas, ou seja, se um certo sítio $i$ é vizinho de outro sítio $j$, então o sítio $j$ é vizinho do sítio $i$. Podemos dispor os $n$ sítios em reticulados unidimensionais ou bidimensionais [14].

Redes 1D Enumeramos os sítios de 1 a $n$. Cada sítio $k, k=1,2, \ldots, n$, tem como vizinhos os $N$ mais próximos sítios à direita e os $N$ mais próximos à esquerda. Desta forma definimos o conjunto $\operatorname{Viz}(k)=\{k+i: i=-N, \ldots, N, i \neq 0\}$ para cada $k=1,2, \ldots, n$. O conjunto $V i z(k)$ é o conjunto dos sítios vizinhos ao sítio $k$ (ver Figura 2). Viz(k) é chamado de vizinhança de ordem $N$ do sítio $k$. Denotamos por $|\operatorname{Viz}(k)|$ a cardinalidade deste conjunto, temos $|\operatorname{Viz}(k)|=2 N$.

Redes 2D Enumeramos os $n$ sítios com um par de índices $(k, l), k=1,2, \ldots, m$ e $l=1,2, \ldots, m^{\prime}, m m^{\prime}=n$, formando assim uma rede retangular. Podemos ter agora vários tipos de vizinhaças simétricas. Citamos apenas os mais usados (ver Figura 2). 
Rede 1D

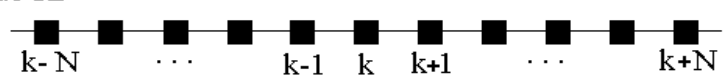

(a)

Rede $2 \mathrm{D}$

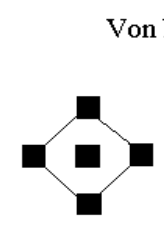

(b) $\mathrm{N}=1$

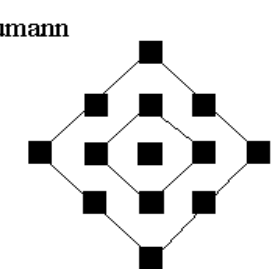

(c) $\mathrm{N}=2$

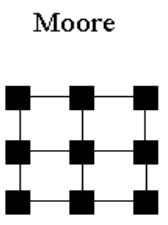

(d) $\mathrm{N}=1$

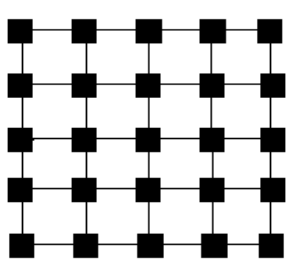

(e) $\mathrm{N}=2$

Figura 2: (a) Vizinhança do sítio $k$ em uma rede unidimensional. (b) Vizinhança do tipo Von Neumann com $N=1$ e (c) $N=2$ em uma rede bidimensional. (d) Vizinhança do tipo Moore com $N=1$ e (e) $N=2$ em uma rede bidimensional.

- Vizinhanças de Von Neumann: definimos os vizinhos do sítio $(k, l)$ através de

$$
\operatorname{Viz}(k, l)=\{(k+i, l+j): 0<|i|+|j| \leq N ;(i, j)\}
$$

Neste caso $|\operatorname{Viz}(k, l)|=2 N(N+1)$.

- Vizinhaças de Moore: os vizinhos de ordem $\mathrm{N}$ do sítio $(k, l)$ forma o conjunto

$$
V i z(k, l)=\{(k+i, l+j):-N \leq i, j \leq N ;(i, j) \neq(0,0)\} .
$$

Consequentemente temos $|V i z(k, l)|=4 N(N+1)$.

O objetivo deste trabalho é determinar a importância do movimento migratório na estabilidade do sistema como um todo. Vamos comparar o sistema global com um de seus fragmentos agindo independentemente. Para isto devemos isolar os efeitos causados pelo movimento migratório. Assim devemos considerar $n$ populações absolutamente idênticas. Mais precisamente, na ausência de migração, cada subpopulação seguiria uma dinâmica temporal dada pela equação (2.1). Mesmo considerando a mesma dinâmica local para todos os sítios, a condição de igualdade entre as subpopulações ainda não está garantida. Isto se deve ao fato de vizinhos mais próximos à fronteira podem ter menos vizinhos em certas direções. Por exemplo em uma rede unidimensional com $N=1$, o primeiro sítio teria apenas 1 vizinho à direita e nenhum à esquerda. Para evitar tais assimetrias, impomos condições de fronteira periódicas, isto é, identificamos as extremidades. Deste modo uma rede unidimentsional será realizada como um anel cíclico e uma rede bidimensional será realizada como uma superfície toroidal.

A dinâmica da metapopulação será dada pela composição de dois processos distintos: a dinâmica local (reprodução e mortalidade) e a dispersão. 
- Dinâmica Local

$$
\begin{aligned}
F & : \mathbb{R}^{n} \rightarrow \mathbb{R}^{n} \\
\left(x^{1}, \ldots, x^{n}\right) & \mapsto\left(f\left(x^{1}\right), \ldots, f\left(x^{n}\right)\right) .
\end{aligned}
$$

- Dispersão

$$
\begin{gathered}
M: \mathbb{R}^{n} \rightarrow \mathbb{R}^{n} \\
\left(x^{1}, \ldots, x^{n}\right) \mapsto\left(M_{1}\left(x^{1}, \ldots, x^{n}\right), \ldots, M_{n}\left(x^{1}, \ldots, x^{n}\right)\right),
\end{gathered}
$$

onde

$$
M_{k}\left(x^{1}, \ldots, x^{n}\right)=\left(1-\mu\left(x^{k}\right)\right) x^{k}+\sum_{j \in V i z(k)} \frac{\mu\left(x^{j}\right) x^{j}}{|V i z(j)|}
$$

Como usamos vizinhanças simétricas, $|V i z(j)|$ é constante e assim podemos suprimir o índice $j$ e escrever simplesmente $|V i z|$. Podemos considerar dois modelos globais inteiramente distintos. O primeiro onde a cada geração a dinâmica local precede à migração. Para isto, definimos $G: \mathbb{R}^{n} \rightarrow \mathbb{R}^{n}$ dada por $G=M \circ F$. Definindo o vetor populacional $x_{t}=\left(x_{t}{ }^{1}, \ldots, x_{t}{ }^{n}\right)^{T}$,onde $x_{t}{ }^{k}$ é o número de indivíduos no sítio $k$ no instante $t$, a dinâmica da metapopulação é dada por

$$
x_{t}=G\left(x_{t}\right)
$$

No segundo modelo a migração precede a dinâmica local. Assim definimos $\tilde{G}=F \circ M$ e a dinâmica global é dada por

$$
x_{t}=\tilde{G}\left(x_{t}\right)
$$

\section{O Estado Homogêneo e sua Estabilidade}

A homogeneidade da população é atingida se o equílibrio homogêneo $\bar{X}=$ $(p, \ldots, p)^{T}, p>0$ é estável. É fácil verificar que para ambas equações (2.4) e (2.5) existe apenas um equilíbrio homogêneo positivo. Além disto suas $n$ componentes coincidem com o equilíbrio do modelo local (sistema desacoplado - equação (2.1)). Resumimos estas informações no seguinte teorema.

Teorema 1 Para ambos os sistemas dados pelas equações (2.4) e (2.5) existe apenas um equilibrio homogêneo não trivial, $\bar{X}=(\bar{x}, \ldots, \bar{x})^{T}, \bar{x}>0$ onde $\bar{x}=f(\bar{x})>0$.

Demonstração: Segue imediatamente das definições que $M \circ F(X)=X \leftrightarrow X=$ $(\bar{x}, \ldots, \bar{x})^{T}$ e de maneira análoga $F \circ M(X)=X \leftrightarrow X=(\bar{x}, \ldots, \bar{x})^{T}$.

A análise da estabilidade de $\bar{X}$ dada pelo Teorema 1 será feita via linearização dos sistemas $(2.4)$ e (2.5) em torno de $\bar{X}$. Os jacobianos $D G(\bar{X})$ e $D \tilde{G}(\bar{X})$ são obtidos no lema a seguir. Definindo a função $\phi$ por $\phi=x \mu(x)$ temos o seguinte lema. 
Lema 1 Seja $\bar{X}$ dado pelo Teorema 1. Então $D G(\bar{X})=D \tilde{G}(\bar{X})=\left[C_{i j}\right]_{n \times n}$, onde

$$
C_{i j}= \begin{cases}f^{\prime}(\bar{x})\left(1-\phi^{\prime}(\bar{x})\right), & i=j \\ \frac{f^{\prime}(\bar{x}) \phi^{\prime}(\bar{x})}{|V i z|}, & j \in \operatorname{Viz}(j) . \\ 0, & \text { c.c. }\end{cases}
$$

Demonstração: Pela regra da Cadeia $D G(X)=D M(F(X)) \cdot D F(X)$ e $D \tilde{G}(X)=$ $D F(M(X)) \cdot D M(X)$. Pelas definições de $M$ e $F$ podemos escrever $D M(\bar{X})=$ $\left[a_{i j}\right]_{n \times n}$, onde

$$
a_{i j}= \begin{cases}1-\phi^{\prime}(\bar{x}), & i=j \\ \frac{\phi^{\prime}(\bar{x})}{|V i z|}, & j \in \operatorname{Viz}(j) \\ 0, & \text { c.c. }\end{cases}
$$

e $D F(\bar{X})=\operatorname{diag}\left(f^{\prime}(\bar{x}), \ldots, f^{\prime}(\bar{x})\right)_{n \times n}$. Note também que $M(\bar{X})=\bar{X}=F(\bar{X})$. Logo $D G(\bar{X})=D M(\bar{X}) \cdot D F(\bar{X})$ e $D \tilde{G}(\bar{X})=D F(\bar{X}) \cdot D M(\bar{X})$. Mas as matrizes $D F(\bar{X})$ e $D M(\bar{X})$ comutam, pois $D F(\bar{X})$ é diagonal com $n$ entradas identicas. Assim $D G(\bar{X})=D \tilde{G}(\bar{X})=D F(\bar{X}) \cdot D M(\bar{X})$ e o resultado segue imediatamente.

Teorema 2 Do ponto de vista da estabilidade do equilíbrio homogêneo positivo dado pelo Teorema 1, os dois sistemas dados pelas equações (2.4) e (2.5) são idênticos, isto é, a ordem dos eventos dinâmica local e migração não afeta a estabilidade do estado homogêneo.

Demonstração: Consequência imediata do Lema 1, já que a estabilidade do equilíbrio homogêneo depende apenas das magnitudes dos autovalores dos Jacobianos no equilíbrio.

O seguinte teorema estabelece alguns resultados importantes na direção do entendimento do papel do acoplamento na estabilidade do equilíbrio homogêneo.

Teorema 3 Se $\phi^{\prime}(\bar{x}) \leq 1$, então o autovalor dominante de $D G(\bar{X})$ (ou de $D \tilde{G}(\bar{X})$ ) é $f^{\prime}(\bar{x})$.

Demonstração: Pelo Teorema 2 é suficiente considerar o sistema dado pela equação (2.4). Pela demonstração do Lema 1 , podemos escrever $D G(\bar{X})=f^{\prime}(\bar{x}) D M(\bar{X})$. Portanto os autovalores de $D G(\bar{x})$ são $f(\bar{x}) \lambda_{1}, \ldots, f(\bar{x}) \lambda_{n}$, onde $\lambda_{1}, \ldots, \lambda_{n}$ são os autovalores de $D M(\bar{x})$. Escrevendo $D M(\bar{X})=\left[a_{i j}\right]_{n \times n}$, os coeficientes $a_{i j}$ são dados pela equação (2.6). Observe que

$$
\sum_{j=1}^{n} a_{i j}=1-\phi^{\prime}(\bar{x})+\sum_{j \in V i z(i)} \frac{\phi^{\prime}(\bar{x})}{|V i z|}=1, \quad \forall i=1,2, \ldots, n .
$$

A hipótese $\phi^{\prime}(\bar{x}) \leq 1$ garante que $a_{i j} \geq 0 \forall i, j=1,2, \ldots, n$. Assim $D M(\bar{X})$ é uma matriz estocástica e portanto $\lambda=1$ é o seu autovalor dominante. Logo $f^{\prime}(\bar{x})$ é o autovalor dominante de $D G(\bar{x})$.

Em relação ao Teorema 3, pode-se observar que 
(1) A hipótese $\phi^{\prime}(\bar{x}) \leq 1$ não é necessária para que $\sum_{j=1}^{n} a_{i, j}=1, \forall i=1,2, \ldots, n$. Portanto ela não é necessária para que $f^{\prime}(\bar{x})$ seja autovalor de $D G(\bar{X})$. Assim, de modo natural, $f^{\prime}(\bar{x})$ ( o autovalor do sistema desacoplado) sempre é autovalor do sistema global. Podemos, portanto, afirmar que o movimento migratório por si só não pode estabilizar uma rede de populações instáveis e independentes.

(2) Um caso particular relevante e freqüentemente estudado [20, 13, 14, 22, 25, 26] é o de fração migratória constante. Neste caso $\phi(x)=\mu x, \operatorname{logo} \phi^{\prime}(x)=\mu \leq 1$ e consequentemente o Teorema 3 é válido.

(3) Os resultados até aqui obtidos independem da dimensionalidade, ou seja é irrelevante a questão de dispormos os sítios como reticulados unidimensionais ou bidimensionais, além disto, no caso bidimensional é irrelevante se consideramos vizinhaças de Von Neumann, Moore ou outra similar. A única restrição é que elas sejam simétricas.

\section{A Instabilidade Causada pelo Movimento Mi- gratório}

Nesta seção mostraremos como a migração pode desestabilizar um sistema de subpopulações estáveis. Tendo em vista o Teorema 3, isto só poderá acontecer se $\phi^{\prime}(x)>1$. Com relação a esta perda da estabilidade via o aumento do valor do parâmetro $\phi^{\prime}(x)$, a dimensão da rede e a geometria das vizinhanças são importantes. Vamos considerar um caso bastante geral: uma rede bidimensional quadrada $n \times n$ com vizinhanças de Moore de ordem $N$. Usando as fórmulas dadas pelo lema, podemos expressar a matriz Jacobiana $D G(\bar{X})$, que neste caso é $n^{2} \times n^{2}$ com estrutura bloco circulante:

$$
D G(\bar{X})=\left[\begin{array}{cccccccc}
A & B & \cdots & B & & B & \cdots & B \\
B & A & B & & \ddots & & \ddots & \vdots \\
\vdots & \ddots & \ddots & \ddots & & \ddots & B & \\
B & & \ddots & \ddots & \ddots & & \ddots & \\
& \ddots & & \ddots & \ddots & \ddots & & B \\
B & & \ddots & & \ddots & \ddots & \ddots & \vdots \\
\vdots & \ddots & & \ddots & & \ddots & \ddots & B \\
B & \cdots & B & & B & \cdots & B & A
\end{array}\right],
$$

onde A e B são matrizes circulantes $n \times n$ 


$$
A=\left[\begin{array}{cccccccc}
a & b & \cdots & b & & b & \cdots & b \\
b & a & b & & \ddots & & \ddots & \vdots \\
\vdots & \ddots & \ddots & \ddots & & \ddots & & b \\
b & & \ddots & \ddots & \ddots & & \ddots & \\
& \ddots & & \ddots & \ddots & \ddots & & b \\
b & & \ddots & & \ddots & \ddots & \ddots & \vdots \\
\vdots & \ddots & & \ddots & & \ddots & \ddots & b \\
b & \cdots & b & & b & \cdots & b & a
\end{array}\right]
$$

$$
B=\left[\begin{array}{cccccccc}
b & b & \cdots & b & & b & \cdots & b \\
b & b & b & & \ddots & & \ddots & \vdots \\
\vdots & \ddots & \ddots & \ddots & & \ddots & & b \\
b & & \ddots & \ddots & \ddots & & \ddots & \\
& \ddots & & \ddots & \ddots & \ddots & & b \\
b & & \ddots & & \ddots & \ddots & \ddots & \vdots \\
\vdots & \ddots & & \ddots & & \ddots & \ddots & b \\
b & \cdots & b & & b & \cdots & b & b
\end{array}\right]
$$

onde $a=f^{\prime}(\bar{x})\left[1-\phi^{\prime}(\bar{x})\right]$ e $b=\frac{f^{\prime}(\bar{x}) \phi^{\prime}(\bar{x})}{4 N(N+1)}$.Em cada linha da matriz $D G(\bar{X})$ há $2 N$ matrizes $B$ e as matrizes $A$ e $B$ possuem $2 N$ elementos $b$ em cada uma de suas linhas. A estrutura peculiar de $D G(\bar{X})$ pode ser usada para a determinação de seus autovalores. O teorema de Friedman [4] torna possível o cálculo dos $n^{2}$ autovalores de $D G(\bar{X})$ (para detalhes ver $[23]), \lambda_{i j}$,

$$
\lambda_{i j}=f^{\prime}(\bar{x})\left[1-\alpha_{i j} \phi^{\prime}(\bar{x})\right], \quad i, j=0,1,2, \ldots, n-1,
$$

onde

$$
\alpha_{i j}=1-\frac{D_{N}\left[\frac{2 \pi i}{n}\right] D_{N}\left[\frac{2 \pi j}{n}\right]}{4 N(N+1)}
$$

$\mathrm{e}$

$$
D_{N}[x]=\frac{\sin x\left[N+\frac{1}{2}\right]}{\sin \frac{x}{2}}
$$

é o núcleo de Dirichlet de ordem $N$. Como $D_{N}[x] \leq 2 N+1$, segue que $0 \leq \alpha_{i j} \leq 2$. O caso extremo $\alpha_{i j}=0$ é obtido quando $i=j=0$ assim $\lambda_{00}=f^{\prime}(\bar{x})$ que é o autovalor local como já era conhecido (ver observação 1 sobre o Teorema 3 ). Note que se $\phi^{\prime}(\bar{x}) \leq 1$ então $\left|1-\alpha_{i j} \phi^{\prime}(\bar{x})\right| \leq 1$ pois $0 \leq \alpha_{i j} \leq 2, \forall i, j=0,1, \ldots, n-1$. Da equação (4.4) seque que $\left|\lambda_{i j}\right| \leq f^{\prime}(\bar{x})$. Como $\lambda_{00}=f^{\prime}(\bar{x})$, temos que $f^{\prime}(\bar{x})$ é o autovalor dominante. Mas isto apenas confirma o Teorema 3 (válido em condições 
mais gerais). A novidade é que se $\phi^{\prime}(\bar{x})>1$, a expressão $\left|1-\alpha_{i j} \phi^{\prime}(\bar{x})\right|$ pode tornarse maior do que a unidade para algum par $\left(i_{\max }, j_{\max }\right) \in 1,2, \ldots, n-1$. Portanto $\left|\lambda_{i_{\max } j_{\max }}\right|>\left|f^{\prime}(\bar{x})\right|$, ou seja o autovalor local não é mais o dominante e além disto, dependendo da magnitude de $\left|1-\alpha_{i_{\max } j_{\max }} \phi^{\prime}(\bar{x})\right|$, podemos ter $\left|\lambda_{i_{\max } j_{\max }}\right|>1$ e $\left|f^{\prime}(\bar{x})\right|<1$ simultaneamente. Isto quer dizer que a instabilidade da rede de subpopulações pode ser causada pelo acoplamento via movimento migratório. Por exemplo, se $N=1$ e $n$ é par, podemos usar as equações (4.4),(4.5) para concluir que $\max _{1 \leq j \leq n-1}\left|1-\alpha_{i j} \phi^{\prime}(\bar{x})\right|>1$ se $\phi^{\prime}(\bar{x})>\frac{4}{3}$. Além disso, $\frac{4}{3}$ é o valor mínimo que o parâmetro $\phi^{\prime}(\bar{x})$ deve ter para haver a possibilidade de instabilidade causada pelo acoplamento. O exemplo seguinte mostra que o Teorema 3 não pode ser melhorado sem um conhecimento maior da dimensão da rede. Considere uma rede unidimensional com $N=1$ e $n$ par. Procedendo de maneira semelhante ao exemplo anterior podemos obter os autovalores de $D G(\bar{X})$,

$$
\lambda_{i j}=f^{\prime}(\bar{x})\left[1-\phi^{\prime}(\bar{x})\left(1-\cos \frac{2 \pi j}{n}\right)\right], \quad i, j=0,1,2, \ldots, n-1 .
$$

Mas $\max _{1 \leq j \leq n-1}\left(1-\cos \frac{2 \pi j}{n}\right)=2$, ocorrendo quando $j=\frac{n}{2}$. Assim o equilíbrio homogêneo é estável se e somente se $\left|f^{\prime}(\bar{x})\right|<1$ e $\left|1-2 \phi^{\prime}(\bar{x})\right|\left|f^{\prime}(\bar{x})\right|<1$. Logo, se $\phi^{\prime}(\bar{x})>1$ a condição $\left|1-2 \phi^{\prime}(\bar{x})\right|>1$ é satisfeita e portanto pode haver instabilidade causada pelo movimento migratório.

\section{Referências}

[1] J.C. Allen, W. M. Schaffer and D. Rosko, Chaos reduces species extinction by amplifying local population noise, Nature, 364 (1993), 229-232.

[2] H. N. Commins, M. P. Hassel and R. M. May, The spatial dynamics of host parasitoid systems, J. Anim. Ecol., 61 (1992), 735-748.

[3] M. Doebeli, Dispersal and dynamics, Theor. Pop. Biol., 47 (1995), 82-106.

[4] B. Friedman, Eigenvalues of Composite Matrices, Proc. Cambr. Phil. Soc., 57 (1961), 37-49.

[5] M.E. Gilpin and I. Hanslki, "Metapopulation dynamics: Empirical and Theoretical Investigations", Academic Press, London, 1991.

[6] C. Grebogi, E. Ott and J. A. Yorke, Chaos, Strange Attractors and Fractal Basin Boundaries en Nonlinear Dynamics, Science, 238 (1987), 632-637.

[7] I. Hanski, "Metapopulation dynamics: from concepts and observations to predictive models", Academic Press, 1997.

[8] I. Hanski and M.E. Gilpin, "Metapopulation Biology: Ecology Genetics and Evolution", Academic Press, 1997.

[9] M.P Hassel, H.N. Commins and R.M. May, The spatial dynamics of host parasitoid systems, J. Anim. Ecol., 61 (1992), 735-748. 
[10] M.P Hassel, O. Miramontes, P. Rohani and R.M. May, Appropriate formulations for dispersal in spatially structured models: comments on Bascompte \& Solé, J. Anim. Ecol., 64 (1995), 662-664.

[11] A. Hastings, Age dependent dispersal is not a simple process: density dependence, stability, and chaos, Theor. Pop. Biol., 41 (1992), 388-400.

[12] A. Hastings, Complex Interactions between dispersal and dynamics: lessons from coupled logistic equations, Ecology, 74 No.5 (1993), 1362-1372.

[13] K. Kaneko, Pattern dynamics in spatiotemporal chaos, Physica D, 34 (1989), $1-41$.

[14] K. Kaneko, Spatiotemporal chaos in one- and two-dimensional coupled map lattices, Physica D, 37 (1989), 60-82.

[15] K. Kaneko, "Theory and Applications of Coupled Map Lattices", Wiley \& Sons, 1993.

[16] A.L. Lloyd, The coupled logistic map: a simple model for the effects of spatial heterogeneity on population dynamics, Theor. Pop. Biol., 173 (1995), 217-230.

[17] R.M. May, Biological populations with nonoverlapping generations: stable points, stable cycles and chaos, Science, 186 (1974), 645-647.

[18] R.M. May, Simple mathematical models with very complicated dynamics, $\mathrm{Na}$ ture, 261 (1976), 459-469.

[19] R.M. May and G.F. Oster, Bifurcations and dynamic complexity in simple ecological models, Amer. Naturalist, 110 (1976), 573-799.

[20] P. Rohani, R.M. May and M.P. Hassel, Metapopulation and equilibrium stability: the effects of spatial structure, J. Theor. Biol., 181 (1996), 97-109.

[21] G.M Ruxton, Density-dependent migration and stability in a system of linked populations, Bull. Math. Biol., 58 (1996), 643-660.

[22] G.M. Ruxton, Synchronization between individuals and the dynamics of linked populations, J. Theor. Biol., 183 (1996), 47-54.

[23] J.A.L. Silva, M.L. de Castro and D.A.R. Justo, Stability in a metapopulation model with density-dependent dispersal. Em preparação.

[24] R.V. Solé and J.P.G. Gamarra, Chaos, dispersal and extinction in coupled ecosystems, J. Theor. Biol., 193 (1998), 539-541.

[25] R.V. Solé and J. Valls, Spiral waves, chaos and multiple attractors in lattice models of interaction populations, Physics Letters A, 166 (1992), 123-128.

[26] R.V. Solé and J. Valls, On structural stability and chaos in biological systems, J. Theor. Biol., 155 (1992), 87-102. 